\title{
Poisson's ratio at 200
}

\section{An understanding of a material's microscopic architecture is important to improve its mechanical properties. Poisson's ratio, which celebrates its bicentenary this year, continues to provide a good metric for that.}

It is the sign of a profound scientific insight if after 200 years a discovery is still the subject of current research. Siméon Denis Poisson's work is no exception. Poisson (see illustration) was an extraordinary scientist. A mathematician and physicist, he is known for Poisson's equation (which describes tensor fields), the Poisson distribution (statistics of random events) and many other phenomena.

This month we celebrate another of Poisson's legacies that to this day remains at the forefront of current research: Poisson's ratio. In 1811, Poisson published his famous book, Traité de Mécanique, on the mechanics of materials, which among other things describes the way materials react to external forces. Take a rubber band. If stretched in one direction, it Poisson's ratio is defined as the ratio of the size change in the direction perpendicular to the applied force versus the expanded length in the direction of the force. The larger the Poisson ratio, the larger the effect: rubber has a larger Poisson ratio than concrete. becomes thinner in the other direction.

Studying a material's Poisson ratio remains an active area of research. There are some materials, such as polymer foams, that have a negative Poisson ratio - if stretched they expand, not contract, in the perpendicular direction. Furthermore, as a macroscopic quantity, Poisson's ratio is directly connected to a material's properties on the microscale. The damage tolerance of some metallic glasses, for example, is directly related to their Poisson ratio. Or more surprisingly perhaps, the fragility of a glass-forming liquid is related to the elastic properties of the solid.

Further details on the role of Poisson's ratio in the research of modern materials are described in the Review Article on page 823. As Neville Greaves and colleagues point out, Poisson's ratio is in no way an outdated quantity. Too many theoretical models underestimate its relevance by setting Poisson's ratio at a fixed value. But Poisson's ratio may not be constant over time at all, for example, when as a result of external stimulus a material changes from being brittle to being ductile.

Although Poisson's legacy is important for the description of a material's

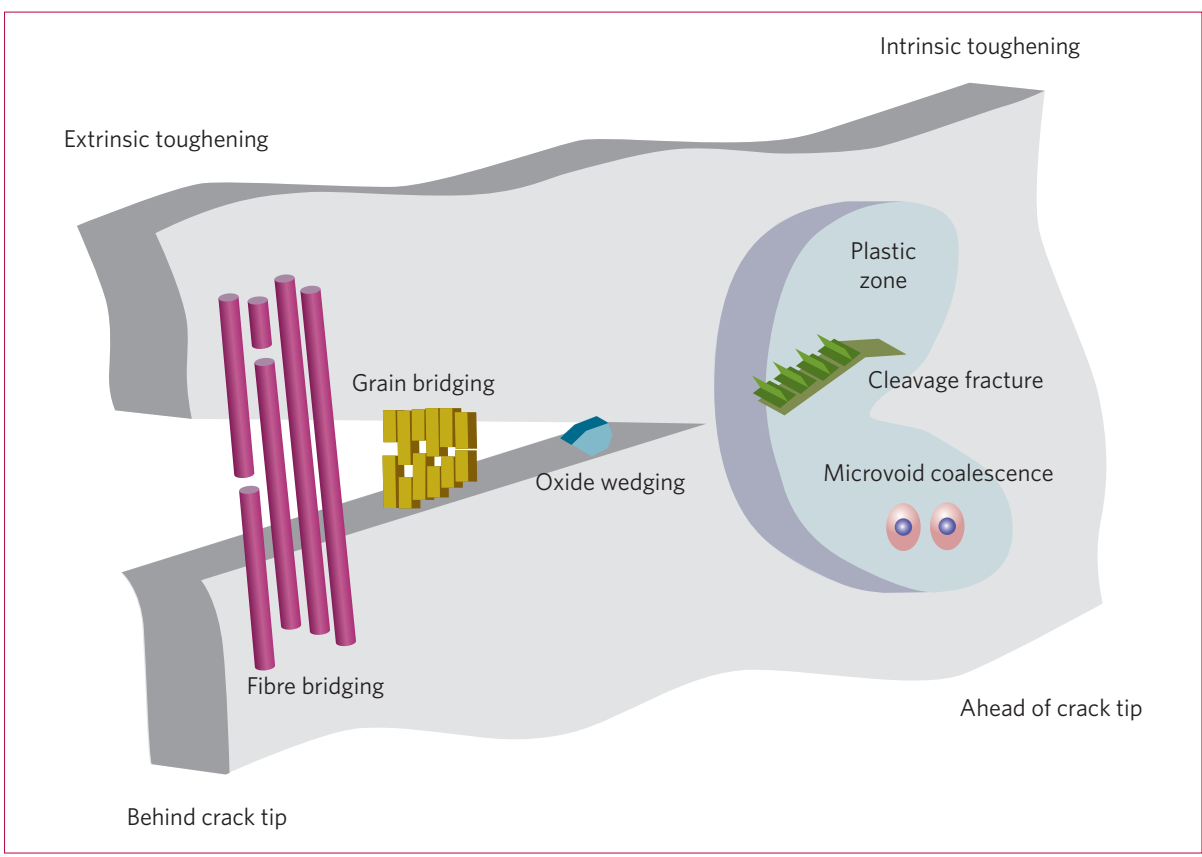

Figure 1 | Materials failure. Intrinsic-damage mechanisms ahead of the crack tip are counterbalanced by extrinsic crack-tip shielding mechanisms.

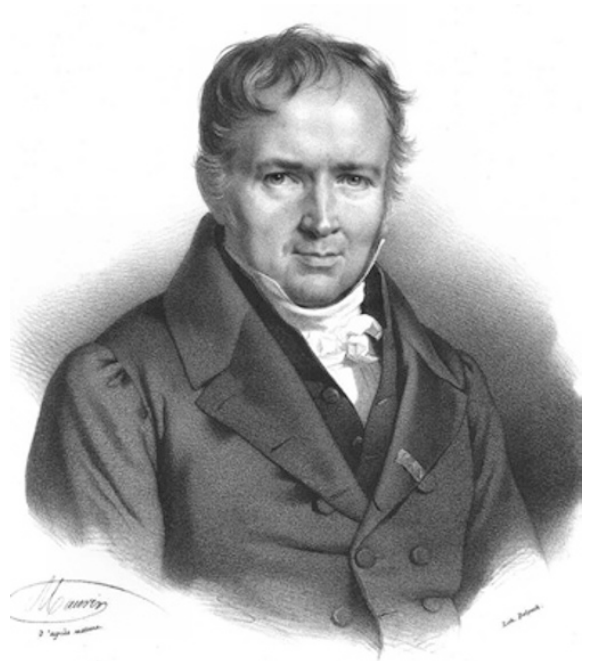

mechanical response more generally, on a microscopic scale it is the understanding and designing of a material's architectural structure on different length scales that is crucial to the development of enhanced materials. In his Progress Article on page 817, Robert Ritchie focuses in particular on the conflicts between strength (hardness) and toughness (resistance to fracture) of materials. Although considered mutually exclusive properties and therefore impossible to optimize at the same time, Ritchie argues that there might be a way out of this dilemma. Properties on different length scales contribute differently to the behaviour of a material. Therefore, a precise control over these different structures could be a successful strategy to enhance properties such as damage tolerance (Fig. 1). Some natural materials such as bone are particularly good examples for the use of such strategies.

Indeed, controlling features on the micro- and nanoscale has already proved a successful strategy, whether to enhance the strength of steels and other alloys, or the mechanical properties of metallic glass composites. Understanding the intricate interplay between properties arising on different length scales undoubtedly will lead to further advances in materials science. And in that process, Poisson's ratio will continue to serve as one of the yardsticks to characterize such new compounds. Poisson would be happy to hear that. 